\title{
HARMONIC MAPPINGS ONTO R-CONVEX DOMAINS
}

\begin{abstract}
The plane domain $D$ is called $R$-convex if $D$ contains each compact set bounded by two shortest sub-arcs of the radius $R$ with endpoints $w_{1}, w_{2} \in D,\left|w_{1}-w_{2}\right| \leqslant 2 R$. In this paper, we prove the conditions of $R$-convexity for images of disks under harmonic sense preserving functions. The coefficient bounds for harmonic mappings of the unit disk onto $R$-convex domains are obtained.
\end{abstract}

Key words: harmonic mappings, R-convex domains, coefficient bounds

2010 Mathematical Subject Classification: 30C45, 30C50, 30C55, 30C99

1. Preliminaries. For a given pair of points $w_{1}, w_{2} \in \mathbb{C}$ such that $\left|w_{1}-w_{2}\right| \leqslant 2 R$ with $0<R<\infty$ we define the $R$-convex hull $E_{R}\left(w_{1}, w_{2}\right)$ of $w_{1}, w_{2}$ as a compact set bounded by the two shortest arcs of the circles of radius $R$ with endpoints $w_{1}, w_{2}$. The set $E_{R}\left(w_{1}, w_{2}\right)$ is strictly convex for each $R>0$ and tends to the segment $\left[w_{1}, w_{2}\right]$ when $R \rightarrow \infty$.

Definition 1. The set $A \subset \mathbb{C}$ is called $R$-convex if $A$ contains each set $E_{R}\left(w_{1}, w_{2}\right)$ provided that $w_{1}, w_{2} \in A$ and $\left|w_{1}-w_{2}\right| \leqslant 2 R$.

It is clear that the Jordan domain $D \subset \mathbb{C}$ is $R$-convex if and only if its closure $\bar{D}$ is $R$-convex. Of course, $R$-convex domains are strictly convex.

$R$-convex sets $\in \mathbb{R}^{n}$ were introduced and studied in $[13,14] . R$-convex sets and domains play an important role in convex analysis and so have applications in many branches of mathematics, physics and economic sciences.

A.W. Goodman [8] defined the convex functions of bounded type independently in the geometrical function theory as an univalent analytic function $h$ in the unit disk $\mathbb{D}=\{z \in \mathbb{C}:|z|<1\}$, such that $\liminf _{|z| \rightarrow 1} k_{h}(z) \geqslant$ $1 / R>0$. Here $k_{h}(z)=\operatorname{Re}\left\{z h^{\prime \prime}(z) / h^{\prime}(z)+1\right\} /\left|z h^{\prime}(z)\right|$ is the curvature of (c) Petrozavodsk State University, 2019 
the image $\Gamma_{r}=h\left(\gamma_{r}\right)$ of the circle $\gamma_{r}=\{z \in \mathbb{D}:|z|=r\}$ under the mapping $h$ at the point $h(z)$. The curvature $k_{h}$ is defined by a standard way as $k_{h}=d \theta / d s$, where $s$ is the natural parameter on $\Gamma_{r}$ and $\theta$ is the argument of the tangent vector to $\Gamma_{r}$.

Let $R \in(0,+\infty)$ be given and $C_{R}$ denote a family of convex analytic functions $h$ in $\mathbb{D}$ of bounded type, such that $h(0)=h^{\prime}(0)-1=0$. These classes were studied by A.W. Goodman $[8,10]$ and K.-J. Wirths [19]. In particular, growth, covering and distortion theorems in $C_{R}$ were proved, as well as coefficient bounds.

Interrelation between $R$-convex domains and analytic univalent functions onto such domains was revealed and investigated in the paper [18] by V. Starkov and N. Shmelev. They proved that for locally univalent analytic function $h$ in $\mathbb{D}$ the domain $D=h(\mathbb{D})$ is $R$-convex if and only if

$$
\operatorname{Re}\left\{z \frac{h^{\prime \prime}(z)}{h^{\prime}(z)}+1\right\} \geqslant \frac{\left|z h^{\prime}(z)\right|}{R} \text { for all } z \in \mathbb{D} \text {. }
$$

More than that, (1) is equivalent to the statement that domains $D_{r}=h\left(\mathbb{D}_{r}\right)$ are $R$-convex for all $r \in(0,1]$, where $\mathbb{D}_{r}=\{z \in \mathbb{D}:|z|<r\}$. So, the heredity property is valid for $R$-convexity of $h\left(\mathbb{D}_{r}\right)$ in the case of analytic functions. Functions $h$ that satisfy condition (1) are univalent.

The theorem of Peschl (cf., [12,19]) claims that if $h \in C_{R}, R>0$, then $k_{h}(z)$ has not local minimums in $\mathbb{D} \backslash\{0\}$ and $k_{h}(z)>1 / R$ in $\mathbb{D} \backslash\{0\}$. Therefore, $h$ is an analytic convex function of bounded type if and only if (1) is true in $\mathbb{D}$ for some $R>0$. This result, together with criterion (1) of $R$-convexity due by V. Starkov and N. Shmelev, immediately leads to

Proposition 1. $C_{R}$ consists of univalent analytic functions $h$ in $\mathbb{D}$ such that $h(0)=h^{\prime}(0)-1=0$ and $h(\mathbb{D})$ is $R$-convex domain.

In this paper, we obtain the conditions of $R$-convexity of $f(\mathbb{D})$ for sensepreserving harmonic functions and prove some estimations and coefficient bounds for the class of normalised univalent harmonic mappings of the disk $\mathbb{D}$ onto $R$-convex domains.

Consider a harmonic and sense-preserving function $f$ in $\mathbb{D}$. It is wellknown (cf., [5]) that every such function $f$ has a form $f=h+\bar{g}$, where $h, g$ are analytic in $\mathbb{D}$ and

$$
h(z)=\sum_{k=0}^{\infty} a_{k} z^{k}, \quad g(z)=\sum_{k=1}^{\infty} b_{k} z^{k} .
$$


The dilatation $\omega(z)=g^{\prime}(z) / h^{\prime}(z)$ of sense-preserving harmonic function $f$ is analytic in $\mathbb{D}$ and $|\omega(z)|<1$ for all $z \in \mathbb{D}$. The tangent vector $\tau(t)$ to curve $\Gamma_{r}=f\left(\gamma_{r}\right), r \in(0,1)$, at the point $f(z), z=r e^{i t}$, has a form

$$
\tau(t)=i\left(z h^{\prime}(z)-\overline{z g^{\prime}(z)}\right) \text { and } \arg \tau(t)=\frac{\pi}{2}+\operatorname{Im} \ln \left(z h^{\prime}(z)-\overline{z g^{\prime}(z)}\right) .
$$

So, direct calculations show that the curvature $k_{f}(z)=(\arg \tau(t))^{\prime} /|\tau(t)|$ of $\Gamma_{r}$ can be computed as

$$
k_{f}(z)=\frac{1}{\left|z h^{\prime}(z)-\overline{z g^{\prime}(z)}\right|} \operatorname{Re}\left\{\frac{z^{2} h^{\prime \prime}(z)+\overline{z^{2} g^{\prime \prime}(z)}+2 \overline{z g^{\prime}(z)}}{z h^{\prime}(z)-\overline{z g^{\prime}(z)}}+1\right\} .
$$

It is clear that harmonic sense-preserving function $f$ should be univalent in $\mathbb{D}_{r}$ if $k_{f}(z) \geqslant 0$ for all $z \in \gamma_{r}$ and domains $D_{r}=f\left(\mathbb{D}_{r}\right)$ will be convex in this case. This is a corollary of the argument principle [5]. It is natural to ask what conditions for the function $f$ guarantee $R$-convexity of $D_{r}$ for $r \in(0,1]$.

2. Conditions of R-convexity. It is well-known (cf., $[3,5]$ ) that harmonic functions $f=h+\bar{g}$ do not possess the heredity property in the case of convexity of $f(\mathbb{D})$. If domain $f(\mathbb{D})$ is convex for a harmonic univalent function $f$ then $f\left(\mathbb{D}_{r}\right)$ can be not convex for all $r \in\left(r_{0}(f), 1\right)$. So we can't expect the $R$-convexity of $f\left(\mathbb{D}_{r}\right)$ when $f(\mathbb{D})$ is $R$-convex and function $f$ is harmonic. The next result describes the $R$-convexity of $f\left(\mathbb{D}_{r}\right)$ in terms of curvature of its boundary.

Theorem 1. Let $f=h+\bar{g}$ be a sense-preserving harmonic mapping of the unit disk $\mathbb{D}$ and $r \in(0,1)$. The domain $D_{r}=f\left(\mathbb{D}_{r}\right)$ is $R$-convex if and only if

$$
\operatorname{Re} \frac{z^{2} h^{\prime \prime}(z)+\overline{z^{2} g^{\prime \prime}(z)}+z h^{\prime}(z)+\overline{z g^{\prime}(z)}}{z h^{\prime}(z)-\overline{z g^{\prime}(z)}} \geqslant \frac{\left|z h^{\prime}(z)-\overline{z g^{\prime}(z)}\right|}{R}
$$

for all $z$ such that $|z|=r$.

Proof. To prove this criterion it is sufficient to note that the arbitrary infinitely-smooth Jordan domain is $R$-convex if and only if the curvature of its boundary is not less than $1 / R$ at every point. This fact was proved in [18] by V. Starkov and N. Shmelev in the course of deriving of the main results. 
In our case, if function $f$ is sense-preserving harmonic in $\mathbb{D}$, then the curve $\partial f\left(\mathbb{D}_{r}\right)$ is infinitely smooth for any $r \in(0,1)$ and $k_{f}$ is defined on $|z|=r$. The condition of the Theorem 1 allows us to state that, if domain $D_{r}=f\left(\mathbb{D}_{r}\right)$ is $R$-convex, then $k_{f}(z) \geqslant 1 / R$ for all $z,|z|=r$. And vice versa, if $k_{f}(z) \geqslant 1 / R$ for $|z|=r$, then $k_{f}$ is positive, so $f$ is univalent convex in $\mathbb{D}_{r}$ and $D_{r}$ is Jordan and $R$-convex. Then formula (3) provides us desired criterion of $R$-convexity of $D_{r}$.

The natural question is to describe $R$-convexity of an open domain $D=f(\mathbb{D})$ in terms of harmonic mappings onto this domain. The sufficient condition of $R$-convexity is given by

Theorem 2. Let $f=h+\bar{g}$ be a sense-preserving harmonic mapping of the unit disk $\mathbb{D}$. The domain $D=f(\mathbb{D})$ is $R$-convex if

$$
\liminf _{|z| \rightarrow 1} k_{f}(z) \geqslant \frac{1}{R}
$$

where $k_{f}$ is given by (3).

Proof. It was proved in [18] that if domain $D$ is $\tilde{R}$-convex for any $\tilde{R}>R$, then $D$ is $R$-convex. Let a harmonic function $f$ be sense-preserving in the unit disk $\mathbb{D}$ and satisfy condition (4) for some $R>0$, but domain $D=f(\mathbb{D})$ be not $R$-convex. Then there exists $\varepsilon>0$ such that $D$ is not $R_{\varepsilon}$-convex, where $R_{\varepsilon}=R+\varepsilon$. Hence, there exists a pair of points $w_{1}, w_{2} \in D$ such that $\left|w_{1}-w_{2}\right| \leqslant 2 R_{\varepsilon}$ and convex hull $E_{R_{\varepsilon}}\left(w_{1}, w_{2}\right) \not \subset D$.

From the other side, condition (4) means that for any $\varepsilon>0$ we can find $r_{\varepsilon} \in(0,1)$ such that $k_{f}(z) \geqslant 1 / R_{\varepsilon}$ on the whole circle $\gamma_{r}=\{z \in \mathbb{D}:|z|=r\}$ for any $r \in\left(r_{\varepsilon}, 1\right)$. So, the curvature of the image $f\left(\gamma_{r}\right)$ of the circle $\gamma_{r}$ is not less than $1 / R_{\varepsilon}$. Theorem 1 implies that $D_{r}=f\left(\mathbb{D}_{r}\right)$ are $R_{\varepsilon}$-convex for all $r \in\left(r_{\varepsilon}, 1\right)$. Hence, the harmonic function $f$ is sense-preserving and convex in all such $\mathbb{D}_{r}$ and, therefore, $f$ is univalent in $\mathbb{D}$. It is clear that $D=\cup_{r \in\left(r_{\varepsilon}, 1\right)} D_{r}$. So, both points $w_{1}, w_{2}$ belong to $D_{r}$ for all sufficiently large $r \in\left(r_{\varepsilon}, 1\right)$. The $R_{\varepsilon}$-convexity of domains $D_{r}$ for such $r$ implies that $E_{R_{\varepsilon}}\left(w_{1}, w_{2}\right) \subset D_{r} \subset D$ in contradiction with assumption $E_{R_{\varepsilon}}\left(w_{1}, w_{2}\right) \not \subset D$. Therefore, domain $D$ is $R$-convex.

Remark. The converse statement to Theorem 2 is not true. Even for the harmonic sense-preserving automorphisms of the unit disk $\mathbb{D}$ the values

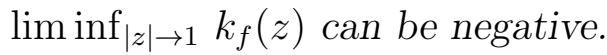


To illustrate this remark we consider function

$$
\theta(t)=\left\{\begin{array}{l}
2 t, \text { for } t \in(0, \pi), \\
0, \text { for } t \in[-\pi, 0] .
\end{array}\right.
$$

This function induces the continuous mapping $w\left(e^{i t}\right)=e^{i \theta(t)}$ of the unit circle onto itself such that $w\left(e^{i t}\right)$ runs once monotonically (but not strictly monotonically) unit circle while $t$ runs from $-\pi$ to $\pi$. It is known from Radó-Knezer-Choquet theorem [5] that the Poisson integral

$$
f_{\theta}(z)=\frac{1}{2 \pi} \int_{-\pi}^{\pi} \frac{1-|z|^{2}}{\left|e^{i t}-z\right|^{2}} e^{i \theta(t)} d t
$$

defines univalent harmonic mapping of $\mathbb{D}$ onto itself with boundary function $e^{i \theta(t)}$. The boundary behaviour of $f_{\theta}$ is such that closed lower half of the unit circle corresponds to the single point 1 , while open upper half of the unit circle is mapped onto whole circle without point 1 . The image of polar grid in $\mathbb{D}$ under mapping $f_{\theta}$ is presented on the left part of the Fig. 1. Note that the images $f_{\theta}\left(\gamma_{r}\right)$ of circles $\gamma_{r}$ are not convex for sufficiently large $r<1$. The geometrical picture of $f_{\theta}\left(\gamma_{r}\right)$ in the neighbourhood of the point 1 is presented on the right part of Fig 1.
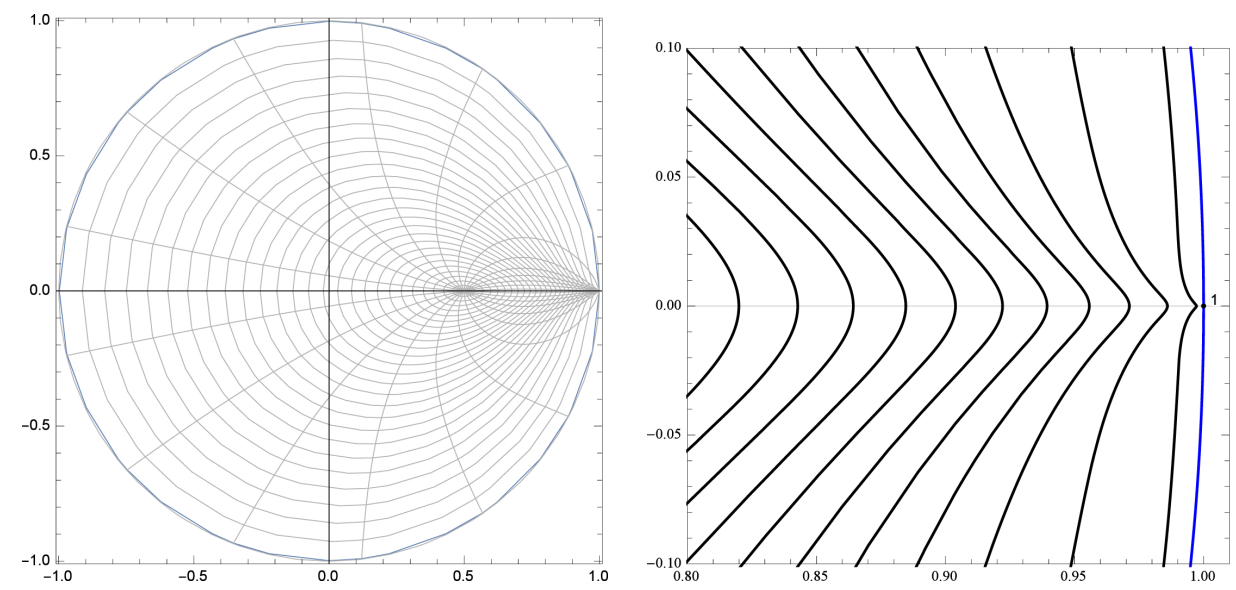

Figure 1: Image of polar grid in $\mathbb{D}$ under mapping $f_{\theta}$ (left). The local nonconvex structure of the images of polar circles $\gamma_{r}$ in the neighbourhood of the point 1 (right).

Using Wolfram Mathematica, it is possible to calculate the curvature $k_{f_{\theta}}(z)$ of $f_{\theta}\left(\gamma_{r}\right)$ at points $z=r e^{i t}$. Let $z=r$ tend from the origin to 1 . 
The image of this radius under $f_{\theta}$ is marked in the left-hand part of Fig. 1 by the bold line. Fig. 2. illustrates the values $k_{f_{\theta}}(r)$ when $r$ tends to 1 . It can be seen from this dependence that curvatures $k_{f_{\theta}}(z)$ become negative

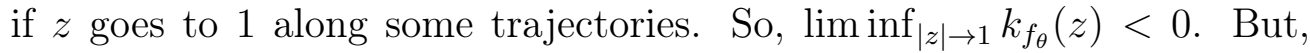
from the other side, it is clear that the unit disk is an $R$-convex domain with $R=1$.

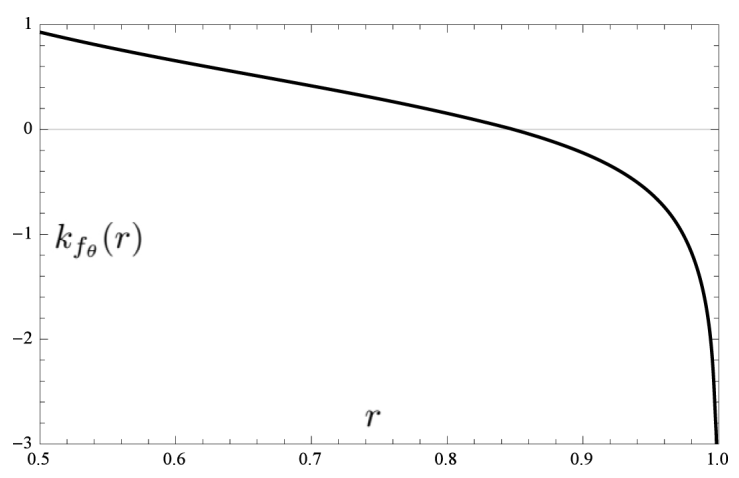

Figure 2: The curvature $k_{f_{\theta}}(r)$ for $r \rightarrow 1-$.

This counter-example leads us to the important fact that in contrast to the analytic case, the set of harmonic univalent functions of the disk $\mathbb{D}$ onto $R$-convex domains is wider than the family of harmonic sensepreserving functions satisfying condition (4).

The next question is to find a maximal radius $r_{0}(R)$ such that sensepreserving harmonic function maps every disk $\mathbb{D}_{r}$ onto $R$-convex domain for all $r \leqslant r_{0}(R)$. It is known [5] that near the origin every $f=h+\bar{g}$ with $h, g$ of form (2) and $\left|b_{1}\right|<\left|a_{1}\right|$ maps infinitesimal disks $|z|<\varepsilon$ onto interiors of convex curves close to the infinitesimal ellipses $\left\{a_{0}+a_{1} z+\overline{b_{1} z}\right.$ : $|z|=\varepsilon\}$. So, $f\left(\mathbb{D}_{\varepsilon}\right)$ should be $R$-convex for sufficiently small $\varepsilon>0$.

The linear hull $L(f)$ of a sense-preserving harmonic function $f=h+\bar{g}$ in $\mathbb{D}$ with $a_{0}=a_{1}-1=0$ is defined as the linear-invariant family of all harmonic sense-preserving functions

$$
f_{\Phi}(z)=\frac{f \circ \Phi(z)-f \circ \Phi(0)}{\Phi^{\prime}(0) \cdot h^{\prime} \circ \Phi(0)}
$$

where $\Phi(z)=e^{i \alpha}(z-\zeta) /(1-\bar{\zeta} z)$ runs over the family of the conformal automorphisms of $\mathbb{D}$.

The affine hull $A(f)$ of a harmonic function $f$ is defined as the family 
of all harmonic sense-preserving functions

$$
f_{\varepsilon}(z)=\frac{f(z)+\varepsilon \overline{f(z)}}{1+\varepsilon b_{1}}
$$

where $\varepsilon$ runs over the disk $\mathbb{D}$.

Let $A L(f):=A(L(f))$ denote the affine and linear hull of $f$. The subfamily $A L^{0}(f) \subset A L(f)$ consists of functions $\tilde{f}$ such that $\tilde{f}_{\bar{z}}(0)=0$. Define $\alpha_{0}=\alpha_{0}(f)=\sup \left|\tilde{f}_{z z}(0)\right| / 2$ and $\beta_{0}=\beta_{0}(f)=\sup \left|\tilde{f}_{\overline{z z}}(0)\right| / 2$, where the suprema are taken over all $\tilde{f} \in A L^{0}(f)$. It is known [3] that $\alpha_{0}$ is finite for all univalent $f$ and $\beta_{0} \leqslant 1 / 2$. The sharp upper bound of $\alpha_{0}$ for an arbitrary univalent harmonic function $f$ is still unknown (though, conjectured) [3]. However, the sharp upper bounds of $\alpha_{0}$ have been obtained for harmonic functions with some special geometric properties (cf., [3,5]). For more results on linear- and affine-invariant families of harmonic functions, see $[6,11,16,17]$.

Theorem 3. Let a sense-preserving harmonic mapping $f=h+\bar{g}$ of the unit disk $\mathbb{D}$ have form (2) and $a_{0}=a_{1}-1=0, \alpha_{0}<\infty$. Then the domain $D_{r}=f\left(\mathbb{D}_{r}\right)$ is $R$-convex for every $r \leqslant r_{0}(R)$, where $r_{0}(R) \in\left(0, \alpha_{0}+\beta_{0}-\sqrt{\left(\alpha_{0}+\beta_{0}\right)^{2}-1}\right)$ is the smallest positive root of the equation

$$
\frac{1-\left|b_{1}\right|}{\left(1+\left|b_{1}\right|\right)^{2}}\left(\frac{1-r}{1+r}\right)^{\alpha_{0}+3 / 2} \frac{r^{2}-2 r\left(\alpha_{0}+\beta_{0}\right)+1}{r}=\frac{1}{R} .
$$

Proof. Let $f$ satisfy the conditions of Theorem 3. The upper and lower bounds of curvature $k_{f}(z)$ of images $f\left(\gamma_{r}\right)$ of the circle $\gamma_{r}, r \in(0,1)$, in the linear- and affine-invariant families of harmonic sense-preserving in $\mathbb{D}$ functions $f$ were published in [11]. In particular, it was proved that $f$ is convex in the disk $|z|<\alpha_{0}+\beta_{0}-\sqrt{\left(\alpha_{0}+\beta_{0}\right)^{2}-1}$ and

$$
k_{f}(z) \geqslant \frac{1-\left|b_{1}\right|}{\left(1+\left|b_{1}\right|\right)^{2}}\left(\frac{1-r}{1+r}\right)^{\alpha_{0}+3 / 2} \frac{r^{2}-2 r\left(\alpha_{0}+\beta_{0}\right)+1}{r}
$$

for all $z$ such that $|z| \leqslant r \leqslant \alpha_{0}+\beta_{0}-\sqrt{\left(\alpha_{0}+\beta_{0}\right)^{2}-1}$ and $\left|b_{1}\right|=\left|f_{\bar{z}}(0)\right|$. The validity of Theorem 3 and condition (5) follows from this result immediately.

The values $\alpha_{0}$ and $\beta_{0}$ are known to be $3 / 2$ and $1 / 2$ if a harmonic function $f$ is convex, i. e., if $f(\mathbb{D})$ is a convex domain. Then Theorem 3 leads to 
Corollary 1. Let $f=h+\bar{g}$ be a sense-preserving harmonic mapping of the unit disk $\mathbb{D}$ onto a convex domain, such that $a_{0}=a_{1}-1=b_{1}=0$. Then the domains $D_{r}=f\left(\mathbb{D}_{r}\right)$ are $R$-convex for every $r \leqslant r_{*}(R) \leqslant 2-\sqrt{3}$, where $r_{*}(R)$ is the smallest positive root of the equation

$$
\left(\frac{1-r}{1+r}\right)^{3} \frac{r^{2}-4 r+1}{r}=\frac{1}{R} .
$$

Note that the radius $r_{*}(R)$ is not the best possible, because every convex harmonic function $f$ such that $a_{0}=a_{1}-1=b_{1}=0$ is convex in any disk $\mathbb{D}_{r}$ for $r \leqslant \sqrt{2}-1$ (cf., [5]) and $2-\sqrt{3}<\sqrt{2}-1$.

3. Coefficient bounds. In this section we introduce the families of normalized univalent harmonic functions onto $R$-convex domains and investigate their properties.

Let $f=h+\bar{g}$, where $h, g$ have form (2), and $f$ be sense-preserving. It is clear that the value $a_{0}=h(0)$ does not influence the radius of $R$-convexity of $f(\mathbb{D})$. Also, if $f$ is harmonic and the domain $f(\mathbb{D})$ is $R$-convex for some $R \in(0,+\infty)$, then $f(\mathbb{D}) / a_{1}$ is $\tilde{R}$-convex for $\tilde{R}=R /\left|a_{1}\right|$. Therefore let us assume that $a_{0}=a_{1}-1=0$.

Definition 2. Let $R \in(0,+\infty)$ be fixed and $C_{H, R}$ denote the set of all harmonic sense-preserving functions in the disk $\mathbb{D}$ such that $f(\mathbb{D})$ is $R$-convex and $a_{0}=a_{1}-1=0$. In addition, $C_{H, R}^{0}$ denotes the subset of $C_{H, R}$ that consists of all $f$ with $b_{1}=0$.

As has been mentioned above, the family of harmonic functions onto $R$-convex domains is wider than the family of harmonic convex functions of bounded type (satisfying inequality (4)). Proposition 1 claims that in the analytic case the family $C_{R}$ of normalized analytic convex function of bounded type consists of analytic mappings onto $R$-convex domains. So, $C_{R} \subset C_{H, R}^{0} \subset C_{H, R}$.

It is known [8] that $C_{R}$ is empty for $R<1$ and $C_{1}$ consists of one element $f \equiv z$ only. In the harmonic case, the same property also holds.

Proposition 2. The family $C_{H, R}$ is empty for any $R<1$. The only member of the family $C_{H, 1}$ is $f \equiv z$.

Proof. As we have mentioned above, every $R$-convex domain $D$ is an image of $\mathbb{D}$ under some analytic convex function of bounded type. It was proved [9] that $D$ is contained in some disk of radius $R$ in this case. 
Therefore, if $f \in C_{H, R}$ then $f(\mathbb{D})$ is strictly convex subdomain of some disk of radius $R$. Then (cf., [5]) $f$ has a continuous boundary function $f\left(e^{i t}\right)=\rho(t) e^{i \theta(t)}$ on $\partial \mathbb{D}$ with continuous real $\rho(t), \theta(t), t \in[0,2 \pi)$; so, the generalisation of the Radó-Knezer-Choquet theorem claims that $f$ is the Poisson integral:

$$
f(z)=c+\frac{1}{2 \pi} \int_{0}^{2 \pi} \frac{1-|z|^{2}}{\left|e^{i t}-z\right|^{2}} \rho(t) e^{i \theta(t)} d t
$$

where $\rho(t) \leqslant R$ for all $t \in[0,2 \pi)$. It is easy to see that

$$
\left|a_{1}\right|=\left|f_{z}(0)\right|=\left|\frac{1}{2 \pi} \int_{0}^{2 \pi} \rho(t) e^{i(\theta(t)-t)} d t\right| \leqslant \frac{1}{2 \pi} \int_{0}^{2 \pi} \rho(t) d t .
$$

Therefore, $\left|a_{1}\right|<1$ if $\rho(t) \leqslant R<1$ and $C_{H, R}$ is empty in this case. If $R=1$, then the equality $\left|a_{1}\right|=1$ implies $\rho(t) \equiv 1$ on $[0,2 \pi)$. Therefore, $F(z)=f(z)-c$ maps the disk $\mathbb{D}$ exactly onto itself, if $a_{1}=1$. It is known [4] that the only harmonic automorphism of $\mathbb{D}$ with $a_{1}=1$ is $F \equiv z$. Thus $f \equiv z+c \equiv z$ because of $f(0)=0$.

The next theorem is an analogue of an area principle for harmonic mappings onto $R$-convex domains.

Theorem 4. Let $f=h+\bar{g} \in C_{H, R}$ where $h, g$ have form (2). Then

$$
2 \sum_{k=1}^{\infty} k^{2}\left|a_{k}\right|^{2} \int_{0}^{1} \frac{r^{2 k-1}\left(1-r^{2}\right)}{\left(1+\left|b_{1}\right| r\right)^{2}} d r \leqslant \frac{R^{2}}{1-\left|b_{1}\right|^{2}} .
$$

Particularly, if $f \in C_{H, R}^{0}$ then

$$
\frac{1}{2}+\sum_{k=2}^{\infty} \frac{k}{k+1}\left|a_{k}\right|^{2} \leqslant R^{2} .
$$

Proof. As we have indicated above [9], every $R$-convex domain $D$ is contained in some disk of radius $R$. Therefore, area of $D=f(\mathbb{D})$ is not greater than $\pi R^{2}$ for any harmonic function $f \in C_{H, R}$. Jacobian of a harmonic function $f$ is equal to $\left|h^{\prime}\right|^{2}-\left|g^{\prime}\right|^{2}$. Also, the dilatation $\omega=g^{\prime} / h^{\prime}$ is analytic in $\mathbb{D}$ and meets the condition of the Schwarz lemma (cf., [7]). Therefore,

$$
\left|\frac{\omega(z)-\omega(0)}{1-\overline{\omega(0)} \omega(z)}\right| \leqslant|z| \text { and }|\omega(z)| \leqslant \frac{|z|+|\omega(0)|}{1+|\omega(0) z|}
$$


as a consequence. For $f \in C_{H, R}$, the coefficient $b_{1}=\omega(0)$ and the following estimations of area of $D$ are true:

$$
\begin{gathered}
\pi R^{2} \geqslant \iint_{D} d u d v=\iint_{\mathbb{D}}\left(\left|h^{\prime}(z)\right|^{2}-\left|g^{\prime}(z)\right|^{2}\right) d x d y= \\
=\iint_{\mathbb{D}}\left|h^{\prime}(z)\right|^{2}\left(1-|\omega(z)|^{2}\right) d x d y \geqslant \iint_{\mathbb{D}}\left|h^{\prime}(z)\right|^{2}\left(1-\left(\frac{|z|+\left|b_{1}\right|}{1+\left|b_{1} z\right|}\right)^{2}\right) d x d y= \\
=\int_{0}^{2 \pi} \int_{0}^{1}\left|h^{\prime}\left(r e^{i t}\right)\right|^{2} \frac{\left(1-\left|b_{1}\right|^{2}\right)\left(1-r^{2}\right)}{\left(1+\left|b_{1}\right| r\right)^{2}} r d r d t= \\
=\left(1-\left|b_{1}\right|^{2}\right) \int_{0}^{1} \frac{r\left(1-r^{2}\right)}{\left(1+\left|b_{1}\right| r\right)^{2}} \int_{0}^{2 \pi}\left|h^{\prime}\left(r e^{i t}\right)\right|^{2} d t d r .
\end{gathered}
$$

For $h(z)=z+\sum_{k=2}^{\infty} a_{k} z^{k}$ the series

$$
\left|h^{\prime}\left(r e^{i t}\right)\right|^{2}=\left|\sum_{k=1}^{\infty} k a_{k} r^{k-1} e^{i(k-1) t}\right|^{2}=\sum_{k, l=1}^{\infty} k l a_{k} \overline{a_{l}} r^{k+l-2} e^{i(k-l) t}
$$

converges uniformly by $t$ for a fixed $r$ as a product of two uniformly converging series $\sum_{k=1}^{\infty} k a_{k} r^{k-1} e^{i(k-1) t}$ and $\sum_{l=1}^{\infty} l \overline{a_{l}}{ }^{l-1} e^{-i(l-1) t}$. The system of exponential functions $\left\{e^{i k t}\right\}$ is orthogonal on $[0,2 \pi]$. Then integration gives

$$
\int_{0}^{2 \pi}\left|h^{\prime}\left(r e^{i t}\right)\right|^{2} d t=\int_{0}^{2 \pi}\left|\sum_{k=1}^{\infty} k a_{k} r^{k-1} e^{i(k-1) t}\right|^{2} d t=2 \pi \sum_{k=1}^{\infty} k^{2}\left|a_{k}\right|^{2} r^{2 k-2} .
$$

Continuing the lower estimation of the area, we obtain:

$$
\pi R^{2} \geqslant 2 \pi\left(1-\left|b_{1}\right|^{2}\right) \sum_{k=1}^{\infty} k^{2}\left|a_{k}\right|^{2} \int_{0}^{1} \frac{r^{2 k-1}\left(1-r^{2}\right)}{\left(1+\left|b_{1}\right| r\right)^{2}} d r
$$

and inequality (6) is proved. If a function $f \in C_{H, R}^{0}$, then $b_{1}=0$ and the second inequality (7) in Theorem 4 follows from (6).

The area theorem allows us to obtain coefficient estimations for harmonic mappings onto $R$-convex domains. 
Corollary 1. If $f \in C_{H, R}^{0}$, then

$$
\left|a_{k}\right| \leqslant\left(\frac{k+1}{k}\left(R^{2}-\frac{1}{2}\right)\right)^{1 / 2} \text { for } k \geqslant 2 .
$$

Indeed, the coefficient bounds for $a_{k}$ follow immediately from (7), because $1 / 2+k /(k+1)\left|a_{k}\right|^{2} \leqslant R^{2}$.

The coefficient problem is one of the most attractive and complicated in the theory of univalent harmonic mappings (cf., [5]). The sharp bound is still not proved even in the case of $\left|a_{2}\right|$ in the family $S_{H}^{0}$ of harmonic univalent mapping from $\mathbb{D}$ into $\mathbb{C}$ such that $a_{0}=a_{1}-1=b_{1}=0$. However, for some special subclasses of $S_{H}^{0}$ the sharp coefficient estimations are known. One of such subclasses was defined as the family $S_{H}^{0}(S)$ of all $f=h+\bar{g} \in S_{H}^{0}$ such that $F=h+e^{i \theta} g \in S$ for some constant $\theta \in \mathbb{R}$. Here $S$ denotes the famous class of univalent analytic functions $F$ in $\mathbb{D}$ such that $F(0)=F^{\prime}(0)-1=0$. Several years ago S. Ponnusamy and A. Sairam Kaliraj [15] obtained the sharp coefficient estimations in $S_{H}^{0}(S)$ and conjectured that $S_{H}^{0}(S)=S_{H}^{0}$. However, recently this conjecture was proved to be wrong [2]. Here we follow the same manner to obtain the coefficient bounds in the analogous subclass of $C_{H, R}^{0}$.

Definition 3. Let $C_{H, R}^{0}\left(C_{R}\right)$ denote the subclass of $C_{H, R}^{0}$ consisting of functions $f=h+\bar{g}$ such that $F=h+e^{i \theta} g \in C_{R}$ for some constant $\theta \in \mathbb{R}$.

K.-J. Wirths [19] obtained the sharp upper bounds for coefficients $A_{2}, A_{3}$ for functions $F(z)=z+A_{2} z^{2}+A_{3} z^{3}+\ldots$ in the families $C_{R}$ of analytic convex functions of bounded type:

$$
\left|A_{2}\right| \leqslant \sqrt{1-\frac{1}{R}},\left|A_{3}\right| \leqslant 1-\frac{1}{R}
$$

Here we prove similar estimations in $C_{H, R}^{0}\left(C_{R}\right)$.

Theorem 5. Let $f=h+\bar{g} \in C_{H, R}^{0}\left(C_{R}\right)$. Then

$$
\left|a_{2}\right| \leqslant \frac{1}{2}+\sqrt{1-\frac{1}{R}},\left|a_{3}\right| \leqslant \frac{4}{3}-\frac{1}{R}+\frac{2}{3} \sqrt{1-\frac{1}{R}} .
$$

Both estimations are sharp when $R \rightarrow \infty$. 
Proof. Analytic and co-analytic parts of harmonic function $f \in C_{H, R}^{0}$ satisfy the equality $g^{\prime}=\omega \cdot h^{\prime}$, where $\omega$ is the dilatation of $f$ and $|\omega|<1, \omega(0)=0$. Let $\omega(z)=\sum_{k=1}^{\infty} c_{k} z^{k}$ in $\mathbb{D}$. Then

$$
\sum_{k=2}^{\infty} k b_{k} z^{k-1}=\left(\sum_{k=1}^{\infty} c_{k} z^{k}\right) \cdot\left(\sum_{k=1}^{\infty} k a_{k} z^{k-1}\right)
$$

where $a_{1}=1$ and, hence, $b_{2}=c_{1} / 2, b_{3}=\left(c_{2}+2 c_{1} a_{2}\right) / 3$. Analytic function $\omega$ meets the conditions of the Schwarz lemma (cf., [7]). Therefore, $\left|c_{1}\right| \leqslant 1$ and $\left|c_{2}\right| \leqslant 1-\left|c_{1}\right|^{2}$ (see [1], for instance).

If $f=h+\bar{g} \in C_{H, R}^{0}\left(C_{R}\right)$, then, by definition, there exists some $\theta \in \mathbb{R}$ such that $F(z)=h(z)+e^{i \theta} g(z) \in C_{R}$. The second coefficient $A_{2}$ of an analytic convex function $F$ of bounded type has the form $A_{2}=a_{2}+e^{i \theta} b_{2}$. Then, using (8), we have

$$
\left|a_{2}\right| \leqslant\left|A_{2}\right|+\left|b_{2}\right| \leqslant\left|A_{2}\right|+\frac{\left|c_{1}\right|}{2} \leqslant \frac{1}{2}+\sqrt{1-\frac{1}{R}} .
$$

To estimate the third coefficient, we note that $A_{3}=a_{3}+e^{i \theta} b_{3}$, where $b_{3}=\left(c_{2}+2 c_{1} a_{2}\right) / 3,\left|a_{2}\right| \leqslant\left|A_{2}\right|+\left|c_{1}\right| / 2$ and $\left|c_{2}\right| \leqslant 1-\left|c_{1}\right|^{2}$. Therefore, applying the second inequality (8), we conclude that

$$
\begin{aligned}
& \left|a_{3}\right| \leqslant\left|A_{3}\right|+\frac{1}{3}\left|c_{2}+2 c_{1} a_{2}\right| \leqslant\left|A_{3}\right|+\frac{1}{3}\left(1-\left|c_{1}\right|^{2}+\left|c_{1}\right|\left(2\left|A_{2}\right|+\left|c_{1}\right|\right)\right)= \\
& =\left|A_{3}\right|+\frac{1}{3}\left(1+2\left|c_{1}\right|\left|A_{2}\right|\right) \leqslant \frac{4}{3}-\frac{1}{R}+\frac{2}{3} \sqrt{1-\frac{1}{R}} .
\end{aligned}
$$

If $R \rightarrow \infty$, then (9) becomes $\left|a_{2}\right| \leqslant 3 / 2$ and $\left|a_{3}\right| \leqslant 2$. This estimations coincide with the known sharp coefficient bounds for the convex harmonic mappings $f$ such that $a_{0}=a_{1}-1=b_{1}=0$. So, the sharpness of (9) for $R \rightarrow \infty$ is proved.

Note that inequalities (9) take the form $\left|a_{2}\right| \leqslant 1 / 2,\left|a_{3}\right| \leqslant 1 / 3$ when $R \rightarrow 1$. There are the best possible estimations [4] in the family of nonnormalised harmonic automorphisms of the unit disk $\mathbb{D}$.

Acknowledgement. The Author expresses gratitude to prof. V. Starkov for fruitful discussion and comments.

This work was supported by the Russian Science Foundation, project 17-11-01229. 


\section{References}

[1] Avkhadiev F. G., Wirths K.-J. Schwarz-Pick type inequalities. Birkhäuser Verlag, Basel, 2009. DOI: https://doi.org/10.1007/ 978-3-0346-0000-2.

[2] Aydogan, M., Bshouty, D., Lyzzaik, A., Sakar F. M. On the shears of univalent harmonic mappings. Complex Anal. Oper. Theory, 2018, published online. DOI: https://doi.org/10.1007/s11785-018-0855-9.

[3] Clunie J., Sheil-Small T. Harmonic univalent functions. Ann. Acad. Sci. Fenn. Math., 1984, vol. 9, pp. 3-25. DOI: https://doi.org/10.5186/ aasfm.1984.0905.

[4] Duren P., Shober G. Linear extremal problems for harmonic mappings of the disk. Proc. Amer. Math. Soc., 1989, 106 (4), pp. $967-973$. DOI: https : //doi.org/10.2307/2047281.

[5] Duren P. Harmonic mappings in the plane. Cambridge, 2004. DOI: https: //doi.org/10.1017/CB09780511546600.

[6] Ganenkova E.G., Starkov V.V. On regularity theorems for linearly invariant families of harmonic functions. Probl. Anal. Issues Anal., 2015, vol. 4 (22), no. 1, pp. 38-56. DOI: https://doi.org/10.15393/j3.art. 2015. 2910.

[7] Goluzin G.M. Geometrical function theory. Moscow, 1966. (in Russian). English version: Goluzin G.M. Geometric theory of functions of a complex variable. Translations of Mathematical Monographs, American Mathematical Society, 1969.

[8] Goodman A.W. Convex functions of bounded type. Proc. Amer. Math. Soc., 1984, vol. 92 (4), pp. 541 -546. DOI: https://doi.org/10.2307/2045423.

[9] Goodman A.W. Convex curves of bounded type. Internat. J. Math. \& Math. Sci., 1985, vol. 8 (4), pp. 625-633. DOI: https://doi.org/10.1155/ S0161171285000680.

[10] Goodman A.W. More on convex functions of bounded type. Proc. Amer. Math. Soc., 1986, vol. 97 (2), pp. 303-306. DOI: https://doi.org/10. $2307 / 2046519$.

[11] Graf S. Yu., Eyelangoli O.R. Differential inequalities in linear- and affineinvariant families of harmonic mappings. Iz. VUZ. Math. (Russian Math.), 2010, vol. 55, no. 10, pp. 69-72 (in Russian). English version: Graf S. Yu., Eyelangoli O.R. Differential inequalities in linear-and affine-invariant families of harmonic mappings. Russian Math. (Iz. VUZ), 2010, vol. 55, no. 10, pp. 60-62. DOI: https://doi.org/10.3103/S1066369X10100075. 
[12] Peschl E. Über die Krümmung von Niveaukurven bei der konformen Abbildung einfachzusammenhängender Gebiete auf das Innere eines Kreises. Math. Ann., 1932, vol. 106 (1), pp. 574-594. DOI: https://doi.org/10. $1007 / \mathrm{BF} 01455902$.

[13] Polovinkin E.S. On strongly convex sets and strongly convex functions. Proc. conf. dedicated to 90-th anniversary of L.S. Pontryagin. Vol. 2, Nonsmooth analysis and optimisation. VINITI, 1999, pp. 66-138. (in Russian)

[14] Polovinkin E.S., Balashov M.V. Elements of convex and strongly convex analysis. Physmathlit, Moscow, 2004. (in Russian)

[15] Ponnusamy S., Sairam Kaliraj A. On the coefficient conjecture of Clunie and Sheil-Small on univalent harmonic mappings. Proc. Indian Acad. Sci., 2015, vol. 125 (3), pp. 277-290. DOI: https://doi.org/10.1007/ s12044-015-0236-5.

[16] Sheil-Small T. Constants for planar harmonic mappings. J. Lond. Math. Soc., 1990, vol. s2-42, pp. 237-248. DOI: https://doi.org/10.1112/ $\mathrm{jlms} / \mathrm{s} 2-42.2 .237$.

[17] Sobczak-Knec M., Starkov V.V., Szynal J. Old and new order of linear invariant family of harmonic mappings and the bound for Jacobian. Ann. Univ. Mariae Curie-Sklodowska. Sect. A., 2011, vol. 65 (2), pp. 191-202. DOI: https://doi.org/10.17951/a.2011.65.2.191-202.

[18] Starkov V.V., Shmelev N.A. Biholomorphic mappings of the disk onto strongly convex domains. Siberian Math. J., 2014, vol. 55, no. 4, pp. 875-881. English version: Starkov V.V., Shmelev N.A. Biholomorphic mappings of the disk onto strongly convex domains. Siberian Math. J., 2014, vol. 55, no. 4, pp. 869-875. DOI: https://doi.org/10.1134/ S0037446614040132.

[19] Wirths K.-J. Coefficient bounds for convex functions of bounded type. Proc. Amer. Math. Soc., 1988, vol. 103 (2), pp. 525-530. DOI: https://doi. org/10.2307/2047174.

Received April 22, 2019.

In revised form, June 6, 2019.

Accepted June 6, 2019.

Published online June 9, 2019.

Tver State University,

33, Zheliabova str., Tver, Russia.

Petrozavodsk State University,

33, Lenina pr., Petrozavodsk, Russia.

E-mail: Sergey.Graf@tversu.ru 